\title{
Síndrome hematofagocítico. Reporte de cuatro casos y revisión de la literatura
}

\author{
PABLO YOUNG ${ }^{1}$, JOSE PERONI ${ }^{1}$, BÁRBARA C. FINN ${ }^{1}$, JULIO E. VENDITTI ${ }^{2}$, \\ VERÓNICA PREITI ${ }^{3}$, EDUARDO BULLORSKY ${ }^{3}$, JULIO E. BRUETMAN ${ }^{1}$.
}

'Servicio de Clínica Médica.

${ }^{2}$ Servicio de Patología.

${ }^{3}$ Servicio de Hematología.

Hospital Británico de Buenos

Aires, Argentina.

Recibido el 15 de marzo de

2010, aceptado el 15 de diciembre de 2010.

Correspondencia a:

Pablo Young

Hospital Británico.

Perdriel 74 (1280)

Buenos Aires, Argentina.

Tel: 541143096400

Fax: 541143043393

E-mail: pabloyoung2003@

yahoo.com.ar

\section{Hemophagocytic syndrome. Report of four cases}

Hemophagocytic syndrome (HS) is a severe hyper inflammatory condition whose cardinal symptoms are prolonged fever, cytopenia, hepatosplenomegaly, and hemophagocytosis by activated, morphologically benign macrophages. The clinical course resembles sepsis, sharing similar physiopathological features. We report four patients with the syndrome. A 61-year-old female presenting with fever and pleuritic pain. During the course of the disease, a pancytopenia was detected and a bone marrow aspiration was suggestive of HS. The patient was treated with cyclosporine and steroids with a good response. A 61-year-old male with fever and pancytopenia and a bone marrow aspirate suggestive of HS. The patient did not respond to treatment and died. A 23-year-old male with fever, pancytopenia and positive Hanta virus antibodies. A bone marrow aspirate was suggestive of HS. The patient recovered without any treatment. A 72-year-old male admitted with the diagnosis of pneumonia, that developed a progressive pancytopenia and bone marrow aspirate was suggestive of HS. A bronchoalveolar lavage showed the presence of Acinetobacter baumanii. Despite treatment with methylprednisolone and gammaglobulin, the patient died. Awareness of the clinical symptoms and of the diagnostic criteria of HS is important to start life-saving therapy in time.

(Rev Med Chile 2011; 139: 224-229).

Key words: Adrenal cortex hormones; Leukopenia; Lymphohisiocytosis.
L a hemofagocitosis puede ser encontrada como un fenómeno aislado en múltiples \lrcorner situaciones como en la anemia hemolítica, enfermedades metabólicas, sepsis y malignidad. El síndrome hemofagocítico reactivo o secundario (SH) o linfohistiocitosis hemofagocítica ( $\mathrm{LHH}$ ) es una entidad clínico-patológica caracterizada por la proliferación sistémica de macrófagos benignos con prominente actividad hemofagocitaria. El SH fue descrito por Chandra y coll $^{1}$ en 1975 en la población adulta, pero fueron Risdall $\mathrm{y} \mathrm{col}^{2}$ en 1979 quienes lo caracterizaron adecuadamente distinguiéndolo de la variante histiocitosis maligna infantil. Se describen cuatro casos internados en el Hospital Británico entre los años 2004 y 2009, y una revisión de la literatura.

\section{Caso 1}

Mujer de 61 años, con antecedentes de hipertensión arterial, hipercolesterolemia, diabetes mellitus tipo 2 no insulino requiriente. Comenzó en diciembre de 2008 con cuadro de mialgias en cinturas escapular y pelviana a predominio proximal más febrícula en contexto de rosuvastatina $20 \mathrm{mg}$ día, asociado a cuadro de odinofagia y cefalea ocasional, a predominio temporal izquierdo, habiendo realizado tratamiento antibiótico con amoxicilina durante una semana con hisopado negativo. Se internó en enero del 2009 dada la persistencia de la sintomatología sumada a fiebre con pirogenemia y dolor pleurítico izquierdo. Su examen físico era normal, sin adenopatías, sin visceromegalias, arterias temporales normales. A nivel del laboratorio, 
el recuento de glóbulos blancos era de 18.800 por $\mathrm{mm} 3$ a predominio polimorfo-nuclear, el hematocrito $38 \%$, recuento plaquetario $418.000 \mathrm{~mm} 3$, creatininemia $0,9 \mathrm{mg} / \mathrm{dl}$, calcemia $8,8 \mathrm{mg} / \mathrm{dl}$, fosfatemia $2,2 \mathrm{mg} / \mathrm{dl}$, uricemia $3 \mathrm{mg} / \mathrm{dl}$, uremia $35 \mathrm{mg} /$ dl, eritrosedimentación (VSG) $102 \mathrm{~mm} / 1$ hora, proteína $\mathrm{C}$ reactiva (PCR) 16 (VN: menor a 0,3 $\mathrm{mg} / \mathrm{dl}$ ), pruebas hepáticas y electrolitos plasmáticos normales. Electroforesis de proteínas con leve aumento de alfa 2. Sedimento de orina normal. El ECG y la radiografía de tórax fueron normales. Se realizaron tres hemocultivos y un urocultivo que fueron negativos. Se realizó una tomografía axial computada (TAC) de cuello, tórax y abdomen que mostró engrosamiento pleural izquierdo con leve derrame pleural, sin tromboembolismo pulmonar y resto sin particularidades. Inició tratamiento empírico con ceftriaxona cubriendo probable foco respiratorio. El ecocardiograma trans-esofágico no mostró vegetaciones. A pesar del tratamiento antibiótico, continuó con registros febriles aislados, por lo cual aproximadamente a los 20 días inició meprednisona $60 \mathrm{mg} /$ día y gammaglobulina intravenosa en dosis de $400 \mathrm{mg} /$ $\mathrm{kg} /$ día por 5 días previo estudios serológicos que se detallan a continuación. Serologías para HIV, sífilis, toxoplasmosis, citomegalovirus (CMV) (serología IgM y antigenemia pp65), Chagas, parvovirus B19, Huddleson, Widal, virus de Epstein Barr (VEB), herpes 6, hepatitis A, B, y C, negativos. Estudios inmunológicos: Factor antinuclear, Latex, anti DNA, anti Ro y La, RNP, Sm, ANCA c y p, anticuerpos anticardiolipina, CH50, C3 y C4 normales o negativos. Otros estudios: Vitamina B12 y fólico normales. A pesar del tratamiento esteroideo continuó con registros febriles a predominio nocturnos y pirogenemia a lo que agregó en la analítica pancitopenia con plaquetopenia 33.000 $\mathrm{mm}^{3}$, anemia con hematocrito $26 \%$, y leucopenia de 2.000 por $\mathrm{mm}^{3}$. Se solicitó ferritina que fue de $2.000 \mu \mathrm{g} / \mathrm{l}$ (VN: hombres 30-400 y mujeres 15125), triglicéridos $301 \mathrm{mg} / \mathrm{dl}$ y fibrinógeno 109\% (normal de 200 a 400\%). Se decidió realizar punción aspiración de medula ósea (PAMO) la cual informó: celularidad medular del 50\%, hiperplasia megacariocítica con cambios dismórficos, se evidencian fenómenos hemofagocíticos (Figura 1). En base a dichos hallazgos se interpretó el cuadro como un $\mathrm{SH}$ secundario a posible causa infecciosa versus autoinmune. Se decidió iniciar tratamiento con ciclosporina $0,2 \mathrm{cc}(200 \mathrm{mg}) \mathrm{c} / 12 \mathrm{~h}$ y rotar la metil-prednisolona a dexametasona $10 \mathrm{mg} \mathrm{c} / 12$ h. La paciente presentó buena respuesta al tratamiento, no constatándose más registros febriles y normalizando el hemograma y las plaquetas. A la semana del egreso se realizó PCR para CMV, siendo esta positiva ( $>5000$ copias; VN: menor de 500 copias) agregándose al tratamiento previo, ganciclovir, con la consiguiente disminución de carga viral y mejoría del cuadro. Se realizó una disminución gradual de esteroides y se suspendió la ciclosporina a los dos meses de iniciada. Se interpretó el cuadro como un $\mathrm{SH}$ secundario a CMV. Luego de 1 año la paciente se encuentra asintomática.

\section{Caso 2}

Hombre de 61 años de edad con antecedente de hipertensión arterial que consultó por astenia, adinamia y fiebre de $38,5^{\circ}$ axilar de dos semanas de evolución, asociado a la aparición de placas eritematosas con áreas de descamación, pruriginosas en arcada inguinal, axilas y en región periumbilical. Ingresa con signos vitales estables, febril, lesiones cutáneas descriptas, hepatomegalia palpable de tres traveses de dedo y esplenomegalia. Sin adenopatías. Se realizó biopsia de piel que mostró prurigo urticariano inespecífico. A nivel del laboratorio el recuento de glóbulos blancos era de 3.000 por $\mathrm{mm}^{3}$ a predominio polimorfonuclear, el hematocrito $29 \%$, recuento plaquetario $95.000 \mathrm{~mm}^{3}$, LDH $9.500 \mathrm{U} / \mathrm{L}$, ferritina $1.500 \mu \mathrm{g} / \mathrm{l}$, VSG $120 \mathrm{~mm} / 1$ hora, PCR 5,5 mg/dl, fibrinógeno

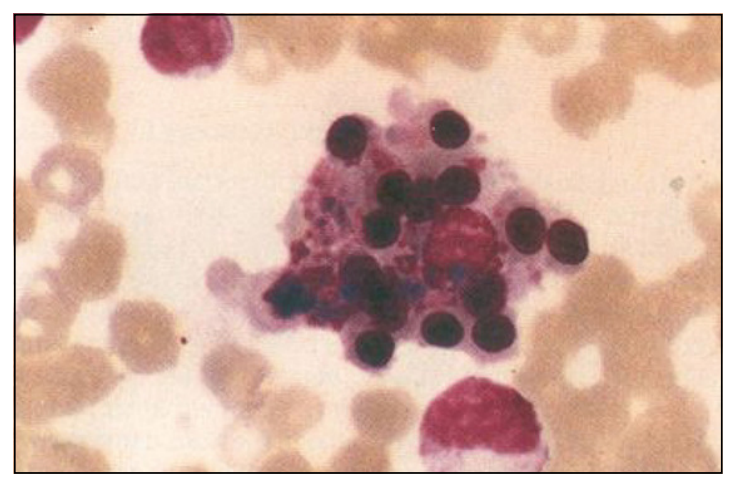

Figura 1. Aspirado de médula ósea con tinción de May Grunwald Giemsa, vista en 100X. Celularidad y progenies conservadas con fenómeno de hemofagocitosis, se observa un macrófago con partículas fagocitadas en su citoplasma y restos nucleares. 
$108 \%$, triglicéridos $450 \mathrm{mg} / \mathrm{dl}$, hepatograma alterado con TGP $376 \mathrm{UI} / \mathrm{ml}$, TGO $125 \mathrm{UI} / \mathrm{ml}$ y FAL $210 \mathrm{mg} / \mathrm{dl}$ (VN: TGP: menor a 30; TGO: menor a 32; FAL: menor a 126). Electroforesis de proteínas con hipoalbuminemia y leve aumento de alfa 2. Sedimento de orina normal. Todas las serologías virales e inmunológicas fueros negativas. Se realizaron cuatro hemocultivos y un urocultivo que fueron negativos. Se realizó una tomografía axial computada de cuello, tórax y abdomen que mostró hepatoesplenomegalia y resto sin particularidades. Se realizó PAMO que mostró hemofagocitosis. Por cumplir seis de los criterios se hace diagnóstico de SH y comenzó terapia con corticoides a altas dosis y gammaglobulina intravenosa en dosis de 400 $\mathrm{mg} / \mathrm{kg} /$ día por 5 días con desaparición de la fiebre. A los días de la administración de la gammaglobulina reapareció la fiebre con incremento de esplenomegalia, por lo que se realizó esplenectomía diagnóstica ante la sospecha de proceso linfo-proliferativo maligno. La biopsia mostró esplenomegalia congestiva, expansión de pulpa roja, hematopoyesis extramedular, ausencia de neoplasia, signos de hemofagocitosis y la biopsia en cuña del hígado esteatosis leve, colestasis mínima y ectasia sinusoidal. Se comenzó terapia con eEtopósido $150 \mathrm{mg} / \mathrm{m}^{2}$. El paciente evolucionó con tricitopenia severa con alto requerimiento transfusional y neutropenia febril prolongada. Nueva PAMO reveló aplasia medular. Desarrolló fallo multiorgánico, coagulopatía por consumo, sangrados masivos y falleció a los 47 días del ingreso.

\section{Caso 3}

Hombre de 23 años que vive en Lobos provincia de Buenos Aires que ingresó por fiebre de tres días de evolución y pancitopenia con plaquetopenia $53.000 \mathrm{~mm}^{3}$, anemia con hematocrito $29 \%$, y leucopenia de 2.500 por $\mathrm{mm}^{3}$. Se realizaron hemocultivos y un urocultivo que fueron negativos. $\mathrm{Al}$ examen físico presentó importante inyección conjuntival y ligera esplenomegalia. Los anticuerpos IgM e IgG para Hanta virus fueron positivos y negativas los de leptospira y fiebre hemorrágica argentina, entre otras. Al tercer día de internación desarrolla insuficiencia respiratoria e infiltrados en vidrio esmerilado en la TAC de tórax con lo cual se hace diagnóstico de síndrome pulmonar por Hanta virus. Se le realizó una PAMO que mostró un aumento de macrófagos en $8 \%$ con hemofagocitosis. Se solicitó ferritina que fue de $7.000 \mu \mathrm{g} / \mathrm{l}$, trigliceridos $401 \mathrm{mg} / \mathrm{dl}$ y fibrinógeno $100 \%$. Con todos estos datos se realizó el diagnóstico de $\mathrm{SH}$ asociado a Hanta virus. A los 10 días de internación sin tratamiento específico normalizó las citopenias $y$ fue dado de alta.

\section{Caso 4}

Hombre de 72 años sin antecedentes de importancia que ingresó por insuficiencia respiratoria y fiebre con diagnóstico de neumonía. Los cultivos y las serologías para virus herpes y CMV fueron negativos, además de todas las serologías al igual que los pacientes previos. Recibió tratamiento antibiótico empírico. A las dos semanas de su ingreso presentó fiebre y empeoramiento de las imágenes radiológicas basales derechas. Se realizó BAL que no mostró crecimiento bacteriano. El paciente desarrolló paulatinamente citopenias de las tres series: $\mathrm{Hb}: 7 \mathrm{~g} / \mathrm{l}$; recuento de leucocitos: 1.600/ $\mathrm{mm}^{3}$; plaquetas: $49.000 / \mathrm{mm}^{3}$. La ferritina fue de $4.000 \mu \mathrm{g} / \mathrm{l}$, trigliceridos $607 \mathrm{mg} / \mathrm{dl}$, fibrinógeno $111 \%$. Se le realizó una PAMO que mostró un aumento de macrófagos en $10 \%$ con hemofagocitosis. Al examen físico solo presentó ligera hepato-esplenomegalia.

Se le administró metilprednisona a una dosis de $1 \mathrm{~g}$ /día por tres días y gammaglobulina en dosis de $400 \mathrm{mg} / \mathrm{kg} /$ día por 5 días. Evolucionó con fiebre persistente y requerimiento de asistencia respiratoria mecánica. Las citopenias no mejoraron. Se repitieron cultivos que siempre fueron negativos, a excepción de un BAL que evidenció el crecimiento significativo de una Acinetobacter baumannii multirresistente que recibió tratamiento. Falleció a los 44 días del ingreso con falla multiorgánica.

\section{Discusión}

Las histiocitosis han sido clasificadas por el grupo de trabajo de la Sociedad Histiocitaria (www.histio.org/society) en 3 grupos: 1) desórdenes relacionados a células dendríticas; 2) desórdenes relacionados a macrófagos y 3 ) desórdenes malignos $^{3}$. El SH pertenece a la segunda categoría, conjuntamente con el síndrome de activación macrofágica (SAM) y la LHH familiar. El término SAM acuñado por Hadchouel y col ${ }^{4}$ en 1985; ha sido usado casi exclusivamente para describir el $\mathrm{SH}$ en asociación con enfermedades reumáticas, especialmente la artritis reumatoidea juvenil ${ }^{5}$. 
El SH puede ser primario o secundario, las formas primarias o genéticas se dividen en dos subgrupos, uno es la familiar o enfermedad de Farquhar y el otro son los síndromes asociados a deficiencias inmunes como el síndrome de Chediak Higashi (Tabla 1). En cuanto a la forma familiar, el $80 \%$ de los casos acurre antes del año de edad. Las formas adquiridas o secundarias son principalmente virales y de ellas el VEB y CMV son los agentes más frecuentes, de las causas malignas el linfoma es la más común (Tabla 1$)^{5,6}$.

El cuadro clínico se caracteriza por fiebre prolongada, hepato-esplenomegalia y citopenias. Las linfadenopatías, el eritema cutáneo, la ictericia y los síntomas neurológicos son menos frecuentes. Típicamente a nivel del laboratorio existe hipertrigliceridemia, niveles de ferritina, transaminasas, bilirrubina, LDH elevados y fibrinógeno bajo ${ }^{6}$.

Los cuatro pacientes presentados en esta comunicación fueron estudiados, pero no tratados en forma uniforme. El diagnóstico fue sospechado luego de descartar otras causas más frecuentes y conocidas. Todos ellos presentaron depleción drástica de las tres series sanguíneas. La fiebre fue el signo presente en todos los pacientes y todos ellos presentaron hemofagocitosis en la primera punción de médula ósea, hallazgo que no es universal, al inicio de la enfermedad. Las infecciones virales fueron los desencadenantes del síndrome

Tabla 1. Causas de SH

\footnotetext{
Primario: familiar, síndrome de Chediak Higashi, Griscelli, Wiskott Aldrich, Di George, enfermedad linfoproliferativa ligada al $X$, inmunodeficiencia combinada severa, inmunodeficiencia común variable, y otros

Secundario:

Virus: como EBV, CMV, HHV-6, HHV-8, HSV, VZV, HIV, Parvovirus, adenovirus, virus hepatotropos.

Otras infecciones: bacterianas, parásitos, espiroquetas y hongos.

Enfermedades reumatológicas: AR juvenil, enfermedad de Still, lupus eritematoso sistémico, esclerodermia, dermatomiosistis, enfermedad de Kawasaki. Neoplasias: linfoma no- Hodgkin y tumores sólidos. Otras: trasplante, post-quimioterapía y tratamiento inmunosupresor, sarcoidosis, enfermedad inflamatoria intestinal, farmacológicas (fenitoina, lamotrigina, metotrexate, sales de oro, vancomicina, trimetroprimasulfametoxazol, sulfazalasina, etarnecept, alentuzumab, BCG intravesical).
}

en 2 pacientes (CMV y Hanta), y en los otros dos casos no se comprobó la causa ${ }^{7}$.

Los criterios diagnósticos utilizados son los establecidos en el protocolo HLH- 2004, el que exige 5 de 8 criterios clínicos, de laboratorio e histopatológicos (Tabla 2$)^{8}$. La fiebre y la esplenomegalia son los criterios clínicos. A las citopenias, se agregan la hipertrigliceridemia $>265 \mathrm{mg} / \mathrm{dl}$ e hipofibrinogenemia $<1,5 \mathrm{~g} / \mathrm{l}$ como criterios de laboratorio. El criterio diagnóstico histopatológico es la confirmación de hemofagocitosis en médula ósea, bazo o ganglios linfáticos. Arbitrariamente se ha adoptado el criterio de Wong y Chang que exige para el diagnóstico que $>2 \%$ de todas las células nucleadas de la médula ósea sean histiocitos con hemofagocitosis?.

Los hallazgos clínicos durante la fase aguda del SH pueden ser explicados como consecuencia de la acción de citoquinas originadas presumiblemente en macrófagos y células $\mathrm{T}$ activadas. La anormalidad inmunológica más frecuente es la alteración global de la función citotóxica. La función de las células NK está marcadamente disminuida o ausente y la actividad citotóxica de los CD8+ también es defectuosa recuperándose luego del tratamiento. Muchos marcadores de la activación de los macrófagos (ferritina, $\beta 2$ microglobulina, enolasa neuronoespecífica) y citoquinas (interferón gamma, factor de necrosis

\section{Tabla 2. Criterios diagnósticos del SH}

1. Enfermedad familiar o defecto genético conocido de SH familiar

2. Presentar 5 de los 8 siguientes:
a. Fiebre
b. Esplenomegalia
c. Citopenia que afecte a dos o más series (hemo- globina $<9 \mathrm{~g} / \mathrm{dL}$, plaquetas $<100,000 \mathrm{~mm}^{3}$, neutrofilos $<1,000 \mathrm{~mm}^{3}$ )
d. Hipertrigliceridemia ( $>265 \mathrm{mg} / \mathrm{dL}$ ) o hipofibrino- genemia (150 mg/dL)
e. Hemofagocitosis en médula ósea, bazo o gan- glios sin evidencia de malignidad
f. Actividad citotóxica de células NK baja o ausente
g. Hiperferritinemia (> $500 \mu \mathrm{g} / \mathrm{l})$
h. SCD25 soluble elevado (IL-2Ra chain; $>2,400 \mathrm{U} / \mathrm{mL}$ ) 
tumoral alfa e interleuquinas) están aumentadas durante el SH. Frecuentemente se detectan altas concentraciones de la cadena alfa del receptor soluble IL-2 (sCD 25), valores superiores a 2.400 $\mu / \mathrm{ml}$ es un nuevo criterio diagnóstico y se asocia con peor pronóstico ${ }^{6,8,10}$.

La sepsis grave se asocia con fallo multiorgánico (FMO). La relación entre SH y FMO no es clara ${ }^{11}$. Reiner reportó 4 casos de FMO entre 23 casos de $\mathrm{SH}^{12}$. Recientemente Kleinert y col. reportaron en nuestro país cuatro casos mortales en el contexto de la terapia intensiva ${ }^{13}$. Ambas entidades comparten desencadenantes infecciosos entre los que se encuentran virus, bacterias, hongos y parásitos. En ambas está presente un nivel aumentado de citoquinas. Si bien esto no es prueba de su vinculación, sugiere que están asociadas a un estado proinflamatorio.

La respuesta al tratamiento es en general peor en los adultos con $\mathrm{SH}$ por razones no conocidas totalmente ${ }^{6}$.

Los objetivos del tratamiento son suprimir la hiperinflamación responsable de los síntomas y destruir a las células infectadas presentadoras de antígeno. Según el protocolo de la Sociedad Histiocitaria revisado en el año 2004 (HLH-2004), la terapeútica inicial consiste en corticoesteroides (CE), preferentemente dexametasona que cruza la barrera hematoencefálica mejor que la prednisolona, ciclosporina A (CSA) y etopósido ${ }^{8}$. Los CE son citotóxicos para los linfocitos e inhiben la expresión de citoquinas, la CSA impide la activación de los linfocitos, el etopósido tiene alta actividad en las enfermedades histiocitarias y es particularmente útil en el SH por VEB. En pacientes con síntomas menos graves, la asociación de CE e inmunoglobulinas puede ser suficiente ${ }^{14}$. Dos grandes series han mostrado resultados promisorios con el uso de gammaglobulina endovenosa (GGEV), especialmente en el SH secundario a infecciones ${ }^{14,15}$. Debe enfatizarse que el tratamiento dirigido solamente contra el patógeno no es suficiente, con la posible excepción de la leishmaniasis, en la cual el tratamiento con anfotericina B suele ser curativo. Tres de nuestros pacientes recibieron GGEV y dos fallecieron sin poder determinar la causa del SH.

Recientemente se ha ensayado con éxito el tratamiento con daclizumab (un anticuerpo monoclonal anti-CD25), en un paciente con SH dependiente de esteroides ${ }^{16}$.

En conclusión creemos que el médico general tiene que tener un alto índice de sospecha, tener en mente los criterios diagnósticos del SH para instaurar a tiempo un tratamiento que salve la vida.

\section{Referencias}

1. Chandra P, Chaudhery SA, Rosner F, Kagen M. Transient histiocytosis with striking phagocytosis of platelets, leukocytes and erythrocytes. Arch Intern Med 1975; 135: 989-91.

2. Risdall RJ, McKenna RW, Nesbit ME, Krivit W, Balfour HH Jr, Simmons RL, et al. Virus associated hemophagocytic syndrome: A benign histyocitic proliferation distinct from malignant histiocytosis. Cancer 1979; 44: 993-02.

3. Favara BE, Feller AC, Pauli M, Jaffe ES, Weiss LM, Arico $\mathrm{M}$, et al. Comtemporary classification of histiocytic disorders. The WHO Committee on Histiocytic/Reticulum cell proliferations. Reclassification Working Group of the Histiocytic Society. Med Pediatr Oncol 1997; 29: 157-66.

4. Hadchouel M, Prieur AM, Griscelli C. Acute hemorrhagic, hepatic and neurologic disease in juvenile rheumatoid arthritis. Possible relationship with drugs or infection. J Pediatr 1985; 106: 561-6.

5. Fukaya S, Yasuda S, Hashimoto T, Oku K, Kataoka H, Horita $\mathrm{T}$, et al. Clinical features of haemophagocytic syndrome in patients with systemic autoimmune diseases: analysis of 30 cases. Rheumatology (Oxford) 2008; 47: 1686-91.

6. Janka GE. Haemophagocytic syndromes. Blood Reviews 2007; 21: 245-53.

7. Lee JJ, Chung IJ, Shin DH, Cho SH, Cho D, Ryang DW, et al. Hemorrhagic Fever with Renal Syndrome Presenting with Hemophagocytic Lymphohistiocytosis. Emerg Infect Dis 2002; 8: 209-10.

8. Henter JI, Horne A, Arico M, et al. HLH-2004: Diagnostic and therapeutic guidelines for hemophagocytic lymphohistiocytosis. Pediatr Blood Cancer 2007; 48: 124-131.

9. Wong KF, Chan JKC. Reactive hemophagocytic syndrome: a clinicopathologic study of 40 patients in an oriental population. Am J Med 1992; 93: 177-80.

10. Egeler RM, Shapiro R, Loechelt B, Filipovich A. Characteristic inmune abnormalities in hemophagocytic lymphohistiocytosis. J Pediatr Hematol Oncol 1996; 18: 340-5.

11. Gauvin F, Toledano B, Champagne J, Lacroix J. Reactive hemophagocytic syndrome presenting as a component 
of multiple organ dysfunction syndrome. Crit Care Med 2000; 28: 3341-45.

12. Reiner AP, Spivak JL. Hematophagic histiocytosis: a report of 23 new patients and a review of the literature. Medicine (Baltimore) 1988; 67:369-88.

13. Kleinert MM, Garate G, Osatnik J, Cicco J, Hunter B, Soria EJ. [Síndrome hemofagocítico reactivo en pacientes graves. Comunicación de 4 casos]. Medicina (Bs As) 2007; 67: 49-52.

14. Larroche C, Bruneel F, Andre MH, Bader-Meunier B, Baruchel A, Tribout B, et al. Intravenously administered gamma-globulins in reactive hemophagocytic syndrome. Multicenter study to assess their importance by the immunoglobulins group of experts of CEDIT of the AP-HP. Ann Med Interne (Paris) 2000; 151: 533-9.

15. Emmenegger U, Schaer DJ, Larroche C, Neftel KA. Haemophagocytic syndromes in adults: current concepts and challenges ahead. Swiss Med Wkly 2005; 135: 299-314.

16. Olin RL, Nichols KE, Naghashpour M, et al. Successful use of the anti-CD25 antibody daclizumab in an adult patient with hemophagocytic lymphohistiocytosis. Am J Hematol 2008; 83: 747-49. 